\section{NUCLEIC ACIDS}

\section{Ready for the Chop}

\section{from our Cell Biology Correspondent}

WHEN the central dogma itself has recently been in the news it is probably wise to fight shy of sweeping generalizations about macromolecular metabolism. But it really secms to be a general rule that the ribosomal RNAs of eukaryotes and prokaryotes are derived from precursor molecules, larger than the mature molecules, by a series of specific cleavages, and messenger RNAs may well arise in a similar way. In eukaryotes including plants mature ribosomal RNAs are cleaved from single large precursor molecules, and although the sizes of the various molecular species gentrated vary somewhat between organisms, the basic maturation process seems to be common to all. The kinetics of the process have been analysed thoroughly in several cell types, but details of the mechanism, the enzymes involved and their intranuclear localization remain for the most part unknown.

A cell free system which supports maturation of ribosomal RNAs would clearly facilitate an analysis of the enzymatic machinery involved, and Hughes and Kafatos (Biochem. Biophys. Res. Commun., 39, 1108; 1970) may have provided one. They claim that maturation of ribosomal RNA proceeds in nuclei isolated from a rather unlikely tissue, developing silk moth wings. In the isolated nuclei $39 \mathrm{~S}$ precursor molecules give rise to $28 \mathrm{~S}$ and $17 \mathrm{~S}$ mature ribosomal RNAs by way of $30 \mathrm{~S}$ and $21 \mathrm{~S}$ immediate precursors. Moreover, preliminary experiments apparently indicate that these changes also occur in nuclear extracts devoid of intact nuclei.

In bacteria, or more precisely in $E$. coli, as Adesnik and Levinthal showed last year (J.Mol. Biol., 46, 281; $1969)$ the $16 \mathrm{~S}$ and $23 \mathrm{~S}$ mature ribosomal RNAs are produced by the cleavage of $17 \mathrm{~S}$ and $24 \mathrm{~S}$ precursor molecules only slightly larger than the mature molecules. Now Jordan, Feunteun and Monier ( $J$. Mol. Biol., 50, 605; 1970) have shown that even the 5S ribosomal RNA has its precursor. In exponentially growing $E$. ccli pulses of ${ }^{32} \mathrm{P}$-phosphoric acid label molecules closely resembling 5S RNA which have the kinetics expected of a precursor to 5S RNA. Comparison of the nucleotide sequences of the precursors and 5S RNA proves their close relationship. The $5^{\prime}$ terminal sequence of $5 \mathrm{~S}$ RNA is pUG, while the precursor molecules have the sequences pAUUUG, pUUUG or pUUG. It is tempting to suggest that the maturation of 5S RNA involves the stepwise removal of the first three bases from the pAUUUG precursor molecules by an exonuclease. Perhaps the final step, conversion of molecules beginning with pUUG to mature 5S RNA, takes place only after the precursor has associated with the ribosomal subunits. As Jordan et al. comment, this might explain why attempts to reconstitute functional $50 \mathrm{~S}$ subunits from protein, 23S RNA and 5S RNA have failed; perhaps only immature 5S RNA can associate with the 50S ribosome.

And what of messenger RNAs? Are they generated by cleavage of high molecular weight precursors? With so little evidence available a categoric answer is unwarranted but there are enough straws in the wind to suggest that the answer may well prove to be yes. Darnell and his associates (Nature, 226; 214; 1970) have argued that mRNAs may be cleaved from the heterogeneous rapidly labelled nuclear RNAs. Now Nissing and Sekeris (Biochim. Biophys. Acta, 209, 484 ; 1970) report that low concentrations of protein isolated from intranuclear ribonucleoprotein particles in rat liver rapidly cleave 50-80S DNA-like RNA into $30 \mathrm{~S}$ pieces. Further incubation results in the formation of $4 \mathrm{~S}$ to $16 \mathrm{~S}$ RNAs. Detailed analysis of these RNAs and the endonuclease which produces them may well throw light on the maturation of messengers.

\section{MUCILAGINOUS SUBSTANCES Adhesive Collaboration}

\section{from a Correspondent}

The Collagen Club joined forces with the Research Panel of the Gelatine and Glue Research Association for a meeting at Birmingham on July 14. The topics discussed, although not of direct interest to collagen investigators, dealt with substances which were intimately associated with connective tissue proteins or with methods involving the use of collagenous material.

Dr J. F. Kennedy (University of Birmingham) surveyed the chemistry of the acidic mucopolysaccharides, pointing out that unusual features had been revealed recently in the primary structure of some of these compounds. For example, certain hitherto unsuspected sugar residues were found to occur irregularly in the polysaccharide chain and these could form linkages with protein chains in protein polysaccharide complexes. He suggested that mucopolysaccharides would act as an adhesive between adjacent polypeptide chains of collagen.

Kennedy described methods for isolating polysaccharide mixtures from tissues and for separating, identifying and estimating the constituents. These methods could be used when very small amounts were available and were valuable for use with pathological tissues. In certain diseases, increased amounts of some mucopolysaccharides were excreted, and their isolation and identification from urine by Kennedy's methods could be useful in diagnosis.

Dr R. Greenshields (University of Aston) was concerned with an industrial product, caramel, a colouring matter produced from sugar syrup, which is added to foods and beverages. Details of its manufacture were usually a closely guarded secret, he said, but a satisfactory material had to fulfil several specific requirements, of which the isoelectric point was partieularly important. Greenshields had devised fairly rapid and reliable methods, based on paper electrophoresis, for obtaining this value. Caramel is a complex mixture of polymeric coloured substances which can be separated on paper chromatograms. He was also investigating the chemistry of the caramel constituents, knowledge, he suggested, that should help to clarify and control the mechanisms of the browning reaction which is so disadvantageous in the food industry.

Mr A. C. Watson and Mr M. Kelly (National Cash Register Co.) discussed microeneapsulation, which is a fairly recent process whereby small particles are coated with a polymer film and then encapsulated by a coacervation process. Gelatine solutions are among suitable media for the latter stage. The product is a free flowing powder with modified properties, useful 